\title{
Vitamin A and gastric cancer risk
}

\section{Levent Filik}

Received: 11 November 2011/ Accepted: 20 December 2011/Published online: 18 January 2012

(C) The International Gastric Cancer Association and The Japanese Gastric Cancer Association 2012

\section{Dear Editor,}

I read with great interest the recent article by Miyazaki et al. [1]. They reported an inverse relationship between gastric cancer development and dietary vitamin A intake. The article describes a very well-designed study and has very important data on this question, which is a matter of debate owing to inconsistent epidemiologic evidence. However, there is another issue to be considered before the conclusion drawn by Miyazaki et al. can be confirmed. Their study has no data related to their cohort's dietary intake of vitamin A before 1988. Gastric cancer has a history of several decades. That is why diet in adolescence or midlife before 40 years of age should also be considered in the study.

\section{Reference}

1. Miyazaki M, Doi Y, Ikeda F, Ninomiya T, Hata J, Uchida K, et al. Dietary vitamin A intake and incidence of gastric cancer in a general Japanese population: the Hisayama Study. Gastric Cancer. doi:10.1007/s10120-011-0092-7.
L. Filik $(\bowtie)$

Gastroenterology Division, Ankara Research Hospital,

Ulucanlar, Altindag, Ankara 06600, Turkey

e-mail: leventfilik@yahoo.co.uk 